\title{
Exclusive hard reactions and QCD
}

\author{
Dieter Mueller*i \\ Institute of Theoretical Physics II, Ruhr-University Bochum, 44780 Bochum, Germany \\ E-mail: dieter.muelleretp2.rub.de
}

\begin{abstract}
Exclusive reactions at large momentum transfer allow to probe the structure of hadrons, in particular of the pion and nucleon, on the amplitude level. They are challenging both the experimental measurements and the theoretical understanding of Quantum Chromodynamics. We shortly introduce the concept of factorization, emphasize the importance of new experimental nucleon and photon-to-pion form factor measurements, and report on phenomenological progress in the description of hard exclusive electroproduction of photons, providing access to generalized parton distribution.
\end{abstract}

35th International Conference of High Energy Physics - ICHEP2010,

July 22-28, 2010

Paris France

\footnotetext{
* Speaker.

${ }^{\dagger}$ An executable for the evaluation of the hard photon electroproduction cross section off proton, fitted to experimental data, is available online: http://calculon.phy.hr/gpd/
} 


\section{Introduction}

Quantum Chromodynamics (QCD) is widely accepted as the Gauge Field Theory that describes the dynamics of constituents that make up hadrons. Historically, this theory grew up from the quark model [W] and the observed scaling behavior in deeply inelastic electron scattering (DIS) [D]. These measurements at the Stanford Linear Accelerator Center showed that the DIS structure function is (nearly) independent on the photon virtuality $\mathscr{Q}^{2}$ []] , which was explained by the incoherent scattering of so-called partons [四, []. These partons where then identified with quarks and gluons, where the underlying gauge symmetry of QCD is SU(3) [6]. It has been immediately realized in perturbation theory that the running QCD fine structure constant $\alpha_{s}\left(Q^{2}\right)$ vanishes for asymptotically large momentum transfer $Q^{2} \rightarrow \infty[\square,[]$. On the other hand there is no experimental evidence so far that quarks exist as free particles, hence, they are confined inside of hadronic matter. This immediately challenges our theoretical capabilities to solve the bound state problems in terms of quark and gluonic degrees of freedom. Since the asymptotic states are given as bound states of the elementary particles in the theory, it is also not straightforward to employ scattering theory. It might be obvious that a QCD understanding is essential for the exploration of new phenomena in high-energy scattering experiments at the Large Hadron Collider, for the understanding of the muon anomalous magnetic moment or the weak-interaction phenomena in B-meson physics.

QCD remains an unsolved theory; however, in the last four decades various tools have been developed to overcome the fundamental challenges. Most important for the QCD phenomenology of middle- and high-energy scattering experiments is the concept of short- and long-distance factorization [[1]]. E.g., the DIS structure function, depending on the Bjorken variable $x_{\mathrm{Bj}}$ and $\mathscr{Q}^{2}$,

$$
F_{2}=x_{\mathrm{Bj}} \sum_{q=u, d, \cdots} \int_{x_{\mathrm{Bj}}}^{1} \frac{d x}{x} C_{q}\left(\frac{x_{\mathrm{Bj}}}{x}, \alpha_{s}\left(\mathscr{Q}^{2}\right)\right) q\left(x, \mathscr{Q}^{2}\right)+\mathscr{O}\left(1 / \mathscr{Q}^{2}\right), C_{q}(z)=e_{q}^{2} \delta(1-z)+\mathscr{O}\left(\alpha_{s}\right),(1.1)
$$

factorizes in perturbatively calculable coefficients $C_{q}$ and parton distribution functions (PDFs) $q$, where $x$ is the longitudinally quark momentum fraction. While in DIS and in inclusive electronpositron scattering one can utilize the optical theorem and relies on the (light-cone) operator product expansion (OPE) of the time-ordered product of two electromagnetic currents, a diagrammatical approach is needed for the description of other inclusive processes such as semi-inclusive deep inelastic scattering, electron-proton annihilation, and inclusive hadron-hadron scattering processes. In such a factorization framework one uses the fact that collinear singularities in a partonic scattering process, which appear after the transverse degrees of freedom are integrated out, are universal and can be absorbed in PDFs. These non-perturbative quantities are process independent, however, they are conventionally defined within a factorization scheme in terms of light-ray operators,

$$
q\left(x, \mu^{2}\right)=\left.\int_{-\infty}^{\infty} \frac{d \lambda}{2 \pi} e^{i \lambda x 2 n \cdot P}\left\langle P\left|\bar{\psi}_{q}(\lambda n) n \cdot \gamma \psi_{q}(-\lambda n)\right| P\right\rangle\right|_{\mu^{2}}, \quad n^{2}=0 \quad(\text { for } n \cdot A=0),
$$

where $\mu^{2}$ denotes the factorization scale that is associated with the hard-scale. The PDFs are extracted from experimental measurements within global fitting, where their evolution w.r.t. $\mu^{2}$ is perturbatively governed by the Dokshitzer-Gribov-Lipatov-Alterelli-Parisi (DGLAP) equations [미, [20, ㄴ]], which read in the quark non-singlet channel:

$$
\mu^{2} \frac{d}{d \mu^{2}} q\left(x, \mu^{2}\right)=\int_{x}^{1} \frac{d y}{y} P\left(x / y, \alpha_{s}\left(\mu^{2}\right)\right) q\left(y, \mu^{2}\right), \quad P(z, \alpha)=\frac{\alpha}{2 \pi} P^{(0)}(z)+\mathscr{O}\left(\alpha^{2}\right) .
$$


The state of the art in the collinear factorization framework, applied to inclusive processes, is now becoming the next-to-next-to-leading (NNLO) approximation, e.g., at this order the DGLAP kernels were evaluated in the pioneering work of Ref. [22, [23]. This might reduce the theoretical uncertainties in the description of inclusive processes to the few percent level or so.

\section{Counting rules, factorization, and perturbative description of exclusive processes}

Often the scaling behavior, observed in experiment, is employed as a criteria to judge on the underlying mechanism. The asymptotic scaling behavior for various exclusive observables was formally derived from (quark) dimensional counting [24, 25] within the assumption that quark binding effects do not induce an additional, i.e., hadronic, scale. These counting rules state, e.g., that the large $s$ behavior of the cross section for an exclusive process $A+B \rightarrow C+D$ behaves as

$$
\frac{d \sigma}{d t}=\frac{1}{s^{n-2}} f(t / s) \quad \text { for } \quad s \rightarrow \infty, \quad t / s-\text { fixed, }
$$

where $n$ is the total number of leptons, photons, and quarks. For the pion, the photon-to-pion transition, the Dirac and Pauli form factors dimensional counting state for $Q^{2}=-t \rightarrow \infty$ :

$$
F^{\gamma \pi \rightarrow \pi}\left(Q^{2}\right) \propto \frac{1}{Q^{2}}, \quad F^{\gamma \gamma^{\star} \rightarrow M}\left(Q^{2}\right) \propto \frac{1}{Q^{2}}, \quad F_{1}^{\gamma^{\star} p \rightarrow p}\left(Q^{2}\right) \propto \frac{1}{Q^{4}}, \quad F_{2}^{\gamma^{\star} p \rightarrow p}\left(Q^{2}\right) \propto \frac{1}{Q^{6}} .
$$

However, already in the early days it is been realized in quark model calculations for wide angle scattering [26] that, e.g., pinch singularities might spoil the dimensional scaling rules (2.

Inspired by the phenomenological success of counting rules and asymptotic freedom, collinear factorization has been also proposed for exclusive processes such as elastic form factors [27, [28], transition form factors, wide angle scattering processes [29], and heavy meson decays [30]. Here, the underlying idea is that the hard probe resolves entirely the partonic content by gluon (or quark) exchanges, which reproduces up to logarithmic corrections the result of dimensional counting. The non-perturbative quantities are now described in terms of meson or baryon distribution amplitudes that are defined to leading twist accuracy in terms of two-body and three-body light ray operators, respectively. For instance, the pion distribution amplitude reads

$$
\phi\left(u, \mu^{2}\right)=\left.\int_{-\infty}^{\infty} \frac{d \lambda}{2 \pi} e^{i \lambda(2 u-1) n \cdot P}\left\langle 0\left|\bar{\psi}(\lambda n) n \cdot \gamma \gamma^{5} \psi(-\lambda n)\right| \pi^{0}(P)\right\rangle\right|_{\mu^{2}}, \quad n^{2}=0
$$

which evolution is governed by the Efremov-Radyushkin-Brodsky-Lepage (ERBL) equation [2], [28]. An alternative mechanism was suggested by Feynman. Here, only the struck parton takes part on the hard reaction while the spectator system remains unresolved. Since of lack of experimental data, a controversial discussion on the underlying mechanism can be found in the literature [3], 12]].

Alternatively, to the collinear factorization framework, it has been proposed to employ a modified factorization scheme, including Sudakov suppression, [33], 34] or to use (light-cone) sum rules [B5]. In particular, for the description of heavy meson decays the effective soft-collinear theory (SCET) has been developed [36]. In all these frameworks the distribution amplitudes, cf. [2.3, are utilized as non-perturbative input, where it is rather popular to take a truncated (collinear) conformal partial wave expansion, which coefficients are given by certain $u$-moments. The first few 

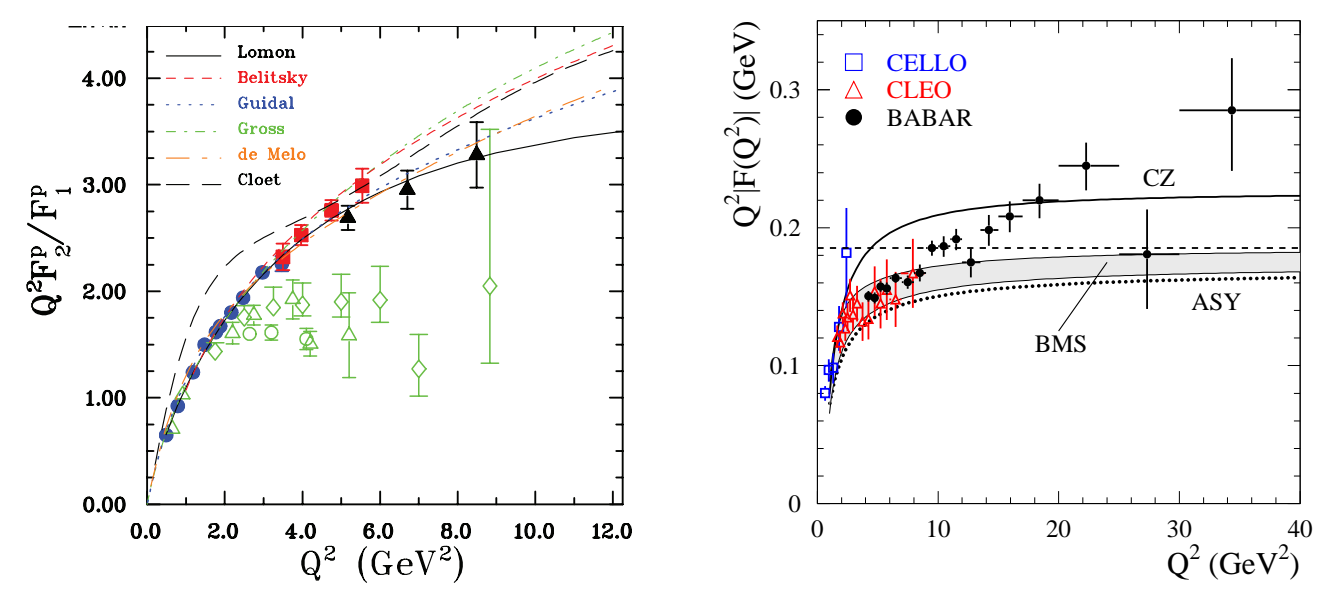

Figure 1: Scaled ratio of proton form factors (left) from Ref. [40] and scaled photon-to-pion transition form factors at large $Q^{2}$ from Ref. [43] (right). For details see the corresponding references.

moments [30] can be evaluated from sum rules or in principle be measured with Lattice QCD, where already the forth moment is hardly to access in present simulations.

Let us now provide two examples for the violation of dimensional scaling at accessible momentum transfer. As displayed in the left panel of Fig. $\square$ by the filled circles, squares and triangles, polarization transfer measurements of the electromagnetic proton form factors up to $Q^{2} \simeq 9 \mathrm{GeV}^{2}$ [37, [38, 39, 40] shows that the ratio $Q^{2} F_{2}\left(Q^{2}\right) / F_{1}\left(Q^{2}\right)$ drastically deviates from a constant behavior, expected from the dimensional counting rules (2.2). For the Pauli form factor $F_{1}\left(Q^{2}\right)$ it is widely assumed that collinear factorization holds true at leading twist, however, so far this is not verified in a NLO calculation. Collinear factorization might be broken for the Dirac form factor $F_{2}\left(Q^{2}\right)$, which is a higher twist quantity [4]]. Pragmatically, the authors of Ref. [42] provided a LO result, where they regularized the non-factorizable end-point singularities. This yields a logarithmical modification, depending on the regularization parameter $\Lambda_{\text {hadron }}$, of the dimensional counting rule,

$$
\frac{Q^{2} F_{2}\left(Q^{2}\right)}{F_{1}\left(Q^{2}\right)} \propto \log ^{2} \frac{Q^{2}}{\Lambda_{\text {hadron }}^{2}},
$$

where $\Lambda_{\text {hadron }}$ can be adjusted to the observed scaling violation (dashed, labeled with Belitsky).

Recently, a striking scaling behavior has been found for the photon-to-pion transition form factor, measured by the BaBar collaboration in electron-positron scattering [4]3]. As shown in the right panel of Fig. 囬, the new BaBar data (filled circles) indicate for $\mathscr{Q}^{2} \gtrsim 10 \mathrm{GeV}^{2}$ a strong deviation from the dimensional counting rule (2.2), which seemed to be compatible with both new $\mathscr{Q}^{2} \lesssim 10 \mathrm{GeV}^{2}$ and previous data (triangle). Note also that an anomalous scaling behavior is not established in the new $\eta$ and $\eta^{\prime}$ transition form factor data from BaBar [44]. Theoretically, the pion transition form factor is the simplest observable that can be obtained from the light-cone OPE,

$$
F^{\gamma^{*} \gamma \rightarrow \pi}\left(\mathscr{Q}^{2}\right)=\frac{2}{3 \mathscr{Q}^{2}} \int_{0}^{1} d u\left[1+\mathscr{O}\left(\alpha_{s}\right)\right] \frac{\phi\left(u, \mathscr{Q}^{2}\right)}{u}+\mathscr{O}\left(1 / \mathscr{Q}^{3}\right) .
$$

In a specific scheme, respecting conformal symmetry, the radiative corrections to the hard scattering amplitude are even known to NNLO accuracy [47], where their size does not indicate a theoretical issue. This form factor was also utilized in a model dependent manner to pin down the pion 

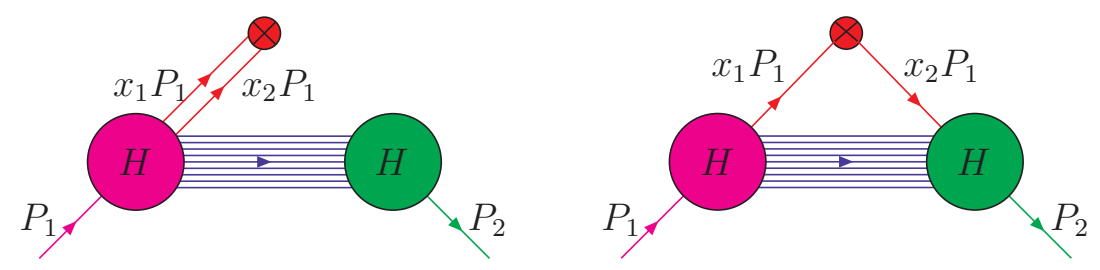

Figure 2: GPDs are associated in the central region $|x| \leq \eta$ with a mesonic-like $t$-channel exchange (left) and in the outer region $\eta \leq|x|$ with a partonic $s$-channel exchange (right).

distribution amplitude [45, 46] (see grayed area in Fig. W). We emphasize that factorization consist of two building blocks, the short distance dynamics which is here, as in (polarized) DIS, controlled by the OPE and the end-point behavior of the non-perturbative pion DA. Various attempts have been given to explain the new BaBar data. The most drastic solution is that the pion DA is almost flat, meaning that factorization does not work, and evolution is not applicable [48], emphasizing the special role of the pion [49]. Other explanations are based on a effective quark mass, leading to a $\log \mathscr{Q}^{2} / m_{\text {eff }}^{2}$ modification [50], sizeable higher-twist contributions [5]], the (updated) inclusion of transverse degrees of freedom [52, [53], or a revised light-cone sum rule analysis [54].

\section{Hard electroproduction of photons and mesons: imaging the nucleon}

Another class of hard exclusive processes is the hard photon and meson electroproduction off nucleon. Here the non-perturbative physics is contained in generalized parton distributions (GPDs) [55], 56, 57]. They are defined by off-forward matrix elements off light-ray operators

$$
q_{S_{1} S_{2}}\left(x, \eta, t, \mu^{2}\right)=\left.\int_{-\infty}^{\infty} \frac{d \lambda}{2 \pi} e^{i \lambda \times n \cdot P}\left\langle S_{2}, P_{2}|\bar{\psi}(\lambda n) n \cdot \gamma \psi(-\lambda n)| P_{1}, S_{1}\right\rangle\right|_{\mu^{2}}, \quad n^{2}=0,
$$

depending on the longitudinal momentum fraction $x$, the skewness $\eta=n \cdot\left(P_{1}-P_{2}\right) / n \cdot\left(P_{1}+P_{2}\right)$, i.e., the longitudinal momentum fraction in the $t$-channel, the momentum transfer square $t$, and the factorization scale $\mu^{2}$. Moreover, one might describe the target spin content within a form factor decomposition $F=\{H, E, \widetilde{H}, \widetilde{E}\}$, where $H(\widetilde{H})$ and $E(\widetilde{E})$ GPDs are the target helicity conserved and flip form factors for a unpolarized (polarized) quark operator (B.W), respectively. In such a description the partonic content of the nucleon is not entirely resolved and so the Feynman mechanism is somehow implemented. For these hard exclusive processes factorization theorems have been proven in the collinear framework at twist-two level [58, 59]. Deeply virtual Compton scattering (DVCS) off nucleon is considered as the theoretically cleanest process. Its amplitude can be parameterized by twelve Compton form factors (CFFs) [6] , which are given in terms of GPDs, e.g., to LO and twist-two accuracy:

$$
\mathscr{F}\left(x_{\mathrm{Bj}}, t, \mathscr{Q}^{2}\right) \stackrel{\mathrm{LO}}{=} \int_{-1}^{1} d x\left[\frac{1}{\xi-x-i \varepsilon} \mp \frac{1}{\xi+x-i \varepsilon}\right] F\left(x, \eta=\xi, t, \mathscr{Q}^{2}\right) .
$$

Here, the Bjorken variable $x_{\mathrm{Bj}}$ might be set equal to $2 \xi /(1+\xi)$. Analogous formulae hold for the LO description of transition form factors (TFFs), measurable in deeply virtual electroproduction of mesons (DVEM) within the exchange of a longitudinally polarized photon. However, the normalization of DVEM amplitudes is also determined by the meson distribution amplitude. The GPD 
evolution equation generalizes both the DGLAG and ERBL one, e.g., in the non-singlet case,

$$
\mu^{2} \frac{d}{d \mu^{2}} F\left(x, \eta, t, \mu^{2}\right)=\int_{-1}^{1} \frac{d y}{\eta} V\left(x / \eta, y / \eta, \alpha_{s}(\mu)\right) F\left(y, \eta, t, \mu^{2}\right) .
$$

All then twist-two kernels are known to NLO accuracy [ $[\mathbb{U}]$. Together with the radiative corrections to the hard-scattering amplitudes [ $[22,[3],[4]$, the collinear formalism is available at NLO accuracy.

Comparing the definitions (‥ $)$ and (B.]), one realizes that GPDs, which are defined on amplitude level, reduces in the forward case to common PDFs. Moreover, the lowest $x$-moment of $H$ and $E$ GPDs provide the definition of (partonic) Pauli and Dirac form factors. Most attention GPDs have received by the fact that their second moment is related to the energy-momentum tensor form factors $A^{q}$ and $B^{q}$, which appear in the proton spin sum rule that has been suggested by X. Ji [57]:

$$
\frac{1}{2}=J^{Q}+J^{G}, \quad J^{Q}=\sum_{q=u, d, \cdots} J^{q}, \quad J^{i}\left(\mathscr{Q}^{2}\right)=A^{i}\left(\mathscr{Q}^{2}\right)+B^{i}\left(\mathscr{Q}^{2}\right) .
$$

Furthermore, it has been realized that GPDs allow for a three-dimensional imaging of nucleons and nuclei $[6]$. In the zero-skewness case the Fourier transform

$$
\rho\left(x, b, \mathscr{Q}^{2}\right)=\frac{\int_{-\infty}^{\infty} d^{2} \vec{\Delta} e^{i \vec{\Delta} \vec{b}} H\left(x, \eta=0, t=-\vec{\Delta}^{2}, \mathscr{Q}^{2}\right)}{\int_{-\infty}^{\infty} d^{2} \vec{\Delta} H\left(x, \eta=0, t=-\vec{\Delta}^{2}, \mathscr{Q}^{2}\right)}
$$

has the probabilistic interpretation to struck a parton in the infinite momentum frame with momentum fraction $x$ and transverse distance $b$ from the proton center [6]]. On the partonic level one might view GPDs as a two-fold object that arises in the central region $|x| \leq \eta$ from a mesonic-like $t$-channel exchange and in the outer region $\eta \leq|x|$ from a partonic $s$-channel view, cf. Fig. $\square$. Both regions are dual to each other, except for a so-called $D$-term [62] that lives entirely in the central region. One another interesting aspect is that GPDs can be represented as the overlap of light-cone wave functions (LCWFs), where the parton number is conserved in the outer, however, not in the inner region. Such a representation allows to establish a model dependent link of GPDs and transverse momentum dependent PDFs. However, it has been understood that a LCWF model has to respect the underlying Lorentz symmetry to obtain a GPD that satisfy polynomiality constraints. If such a model LCWF is at hand, one might be able to restore the whole GPD from the overlap in the outer region [14]. In other words one essentially models the Field Theoretical aspects of QCD, which immediately provides the known challenges: understanding of Regge behavior or whether the generic large $-t$ and $x$ behavior arises from the perturbative [15] or non-perturbative region [123]. In fact, GPDs build up a whole framework for description of hadron structure [63, 64].

Much effort to measure hard exclusive electroproduction processes has been spent in the last decade by the $\mathrm{H} 1$ and ZEUS collaborations (DESY) in the small $x_{\mathrm{Bj}}$ region and at the fixed target experiments HERMES (DESY), CLAS (JLAB), and Hall A (JLAB) in the moderate $x_{\mathrm{Bj}}$ region. The intricate variable dependence of GPDs makes the phenomenology on the first view to a cumbersome task. Indeed, for almost one decade only model predictions were given, which could not reproduce the growing set of DVCS data. For hard meson electroproduction a hand-bag model approach, including transverse degrees of freedom and employing a specific GPD ansatz [65], reproduces experimental data at small and moderate values of $x_{\mathrm{Bj}}$. 


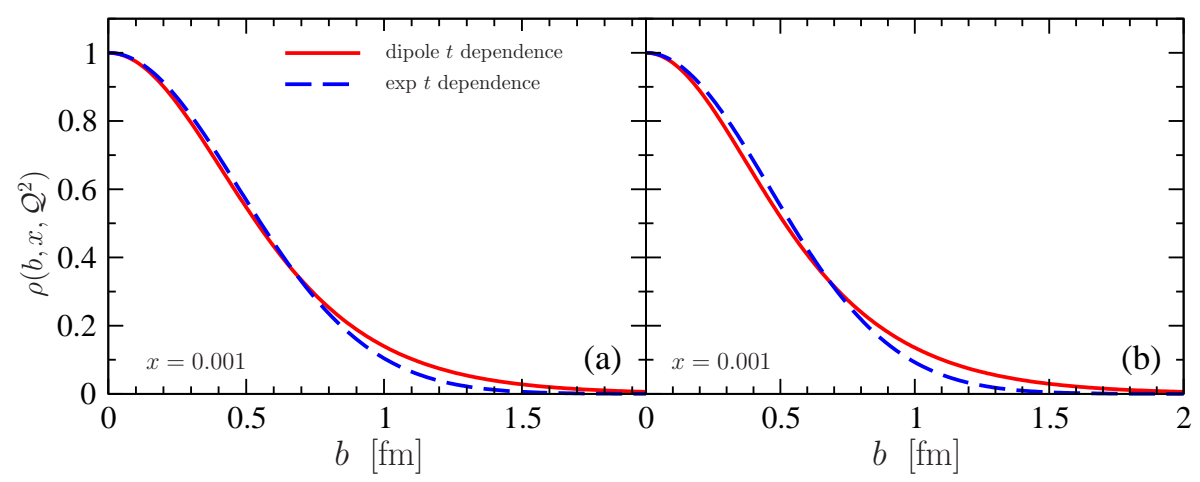

Figure 3: Quark (a) and gluon (b) transverse profile function (B.5]) for $\mathscr{Q}^{2}=4 \mathrm{GeV}^{2}$ and $x=10^{-3}$ is obtained from a six parameter DVCS fit, see Ref. [U]] for details.

Flexible GPD models, based on a Mellin-Barnes integral, were utilized the first time in fits to DVCS at small $x_{\mathrm{Bj}}$ to LO, NLO, and NNLO accuracy, where $\chi^{2} /$ d.o.f. $\approx 1$ [प5]. Here one accesses the DVCS cross section, which is essentially expressed by $\mathscr{H}$ and $\mathscr{E}$ CFFs:

$$
\left.\frac{d \sigma^{\mathrm{DVCS}}}{d t}\left(W, t, \mathscr{Q}^{2}\right) \approx \frac{\pi \alpha^{2}}{\mathscr{Q}^{4}} \frac{W^{2} x_{\mathrm{Bj}}^{2}}{W^{2}+\mathscr{Q}^{2}}\left[|\mathscr{H}|^{2}-\frac{t}{4 M_{p}^{2}}|\mathscr{E}|^{2}\right]\left(x_{\mathrm{Bj}}, t, \mathscr{Q}^{2}\right)\right|_{x_{\mathrm{Bj}} \approx \frac{\mathscr{Q}^{2}}{W^{2}+\mathscr{Q}^{2}}} .
$$

At small $x_{\mathrm{Bj}} \mathscr{H}$ arises from a "pomeron" exchange and the findings might be reparameterized as

$$
\operatorname{Im} \mathscr{H}\left(x_{\mathrm{Bj}}, t, \mathscr{Q}^{2}\right) \sim \pi h\left(t, \mathscr{Q}^{2}\right) x_{\mathrm{Bj}}^{-\alpha\left(\mathscr{Q}^{2}\right)-\alpha^{\prime}\left(\mathscr{Q}^{2}\right) t} .
$$

In accordance with pQCD evolution, one finds that the intercept $\alpha$, which is slightly larger than one at $\mathscr{Q}_{0}^{2}=4 \mathrm{GeV}^{2}$, increases with growing $\mathscr{Q}^{2}$, while the slope parameter $\alpha^{\prime} \sim 0.15 / \mathrm{GeV}^{2}$ is smaller than for a soft pomeron and decreases with growing $\mathscr{Q}^{2}$. The target helicity-flip $\mathscr{E}$ CFF might be associated with the spin-flip "pomeron" [0]]. It might be sizeable, however, in the cross section (B.6) it is suppressed by the factor $\langle\langle|t|\rangle\rangle / 4 M_{p}^{2} \sim 0.05$. Another handle on $\mathscr{H}$ and $\mathscr{E}$ CFFs is the beam charge asymmetry that arises from the interference of Bethe-Heitler and DVCS amplitudes,

$$
A_{\mathrm{BC}}(\phi)=\frac{d \sigma^{+}-d \sigma^{-}}{d \sigma^{+}+d \sigma^{-}} \propto x_{\mathrm{Bj}}\left(F_{1}(t) \Re \mathrm{R} \mathscr{H}-\frac{t}{4 M_{p}^{2}} F_{2}(t) \Re \mathrm{R} \mathscr{E}\right) \cos (\phi)+\cdots
$$

Unfortunately, the experimental errors [प]] do not allow to separate $\mathscr{H}$ and $\mathscr{E}$ contributions. Since of the large lever arm $3 \mathrm{GeV}^{2} \lesssim \mathscr{Q}^{2} \lesssim 80 \mathrm{GeV}^{2}$, evolution can be used to decompose the sea quarks and gluons. The findings for the transverse profiles (B.5) of quarks and gluons are shown in Fig. [].

In fixed target kinematics one might rely on $\mathscr{Q}^{2}$-scaling, where the "dispersion relation", e.g.,

$$
\begin{gathered}
\mathfrak{I m} \mathscr{F}\left(x_{\mathrm{Bj}}, t, \mathscr{Q}^{2}\right) \stackrel{\mathrm{LO}}{=} \pi F\left(\xi, \xi, t, \mathscr{Q}^{2}\right), \quad F=\{H, E, \widetilde{H}, \widetilde{E}\}, \\
\operatorname{Re}\left\{\begin{array}{c}
\mathscr{H} \\
\mathscr{E}
\end{array}\right\}\left(x_{\mathrm{Bj}}, t, \mathscr{Q}^{2}\right) \stackrel{\mathrm{LO}}{=} \mathrm{PV} \int_{0}^{1} d x \frac{2 x}{\xi^{2}-x^{2}}\left\{\begin{array}{c}
H \\
E
\end{array}\right\}\left(x, x, t, \mathscr{Q}^{2}\right) \pm \mathscr{D}\left(t, \mathscr{Q}^{2}\right),
\end{gathered}
$$

offers a simple tool ${ }^{1}$ to access CFFs or GPDs at LO from measurements [미]. Thereby, the integral (B.JO) is taken as a sum rule to access GPDs on the cross-over line, even in the region that is exper-

\footnotetext{
${ }^{1}$ At LO one might view the GPD on the $\eta=x$ cross-over line as "spectral function" (B.. $)$. The GPD support ensures that the real part (B.Td) can be alternatively evaluated from Eq. (B.2), where the subtraction constant $\mathscr{D}$ can be calculated from either $H$ or $E[$ [67, 68, 69]. To pin down the GPD in the outer region $x \geq \eta$, one might utilize evolution (B.3).
} 
imentally not accessible. In these kinematics one uses the interference of Bethe-Heiter and DVCS amplitudes to access various observable, which are approximatively given as a linear combination of CFFs and nucleon form factors. Neglecting, the presumable small transverse photon helicity flip contributions, one can at least in principle access the imaginary and real part of all remaining four twist-two and four twist-three related CFFs. Such a program is only doable if both kinds of polarized electron beams are present and the polarization of the target can be adjusted in longitudinal and both transverse directions. At the HERMES experiment a variety of charge and single spin asymmetries were published for $\left\langle\left\langle x_{\mathrm{Bj}}\right\rangle\right\rangle \sim 0.1$, unfortunately, with rather large uncertainties. The CLAS experiment provided also beam spin asymmetries and the HALL A experiment even sums and differences of electron helicity dependent cross sections. Often, it is thought that all of these observables are dominated by the interference term and by $H$ GPD. However, the magnitude and the steep raise of the unpolarized cross section measurements at $x_{\mathrm{Bj}}=0.36$ from HALL A raise some concern on this assumption. In Fig. 团 we show some fitting results. The solid, dotted and dashdotted lines arise from "dispersion integral" fits, where the small- $x$ behavior of sea-quarks were pinned down from $\mathrm{H} 1$ and ZEUS data and evolved backwards to the scale $\mathscr{Q}^{2} \sim 2 \mathrm{GeV}^{2}$. The gap between these curves somehow quantifies the importance of HALL A data. So far an error estimate has not been given, which is considered as an intricate task. The dotted line is a model prediction, used in the description of hard electroproduction of light vector mesons. Its value overshoots the DVCS results, which, however, in spite of theoretical and experimental uncertainties it cannot be viewed as critical. The filled squares are CFF fits, asking for the value of the real and imaginary part of twist-two CFF, where model dependent bounds has been utilized for the unconstrained degrees of freedom. The large error bars, which size is correlated with the model dependent boundaries, mainly reflect the uncertainty that arise from the limited number of observables. This is also clearly demonstrated by the filled diamonds, where measurements on a longitudinal polarized target have been included and so the size of error bars is drastically reduced. The circles finally arise from the $H$ dominance hypothesis and a GPD model, using a smeared expansion in polynomials. Here the smallness of errors reflects a more tied bound of the unconstrained degrees of freedom. Finally, the triangles arise from a neural network fit within $H$ dominance hypothesis, where only the experimental errors where propagated. We might conclude from the left panel in Fig. 团 that in spite of lack of data, all of the DVCS results are compatible for $0.1<x_{B j}<0.35$, while the right panel clearly reminds us that HALL A cross section measurements remain challenging.

\section{Conclusions}

Pioneering measurements at large $Q^{2}$ revealed unexpected scaling behavior of elastic and transition form factors. These experimental results trigger a renewed debate on the underlying QCD mechanism, including the basic question: Are the constituents entirely resolved in a hard exclusive process? In the hard exclusive electroproduction of photons and mesons this question is irrelevant, since the amplitudes are described here in terms of GPDs that incorporate the Feynman mechanism. These GPDs are now used as a tool to quantify the partonic picture of the nucleon (or other hadrons). Phenomenologically, they are accessed within both the collinear factorization and the hand-bag approach. The growing amount of data calls for a unique description, available in the collinear factorization framework to NLO accuracy, in terms of flexible GPD models, which 

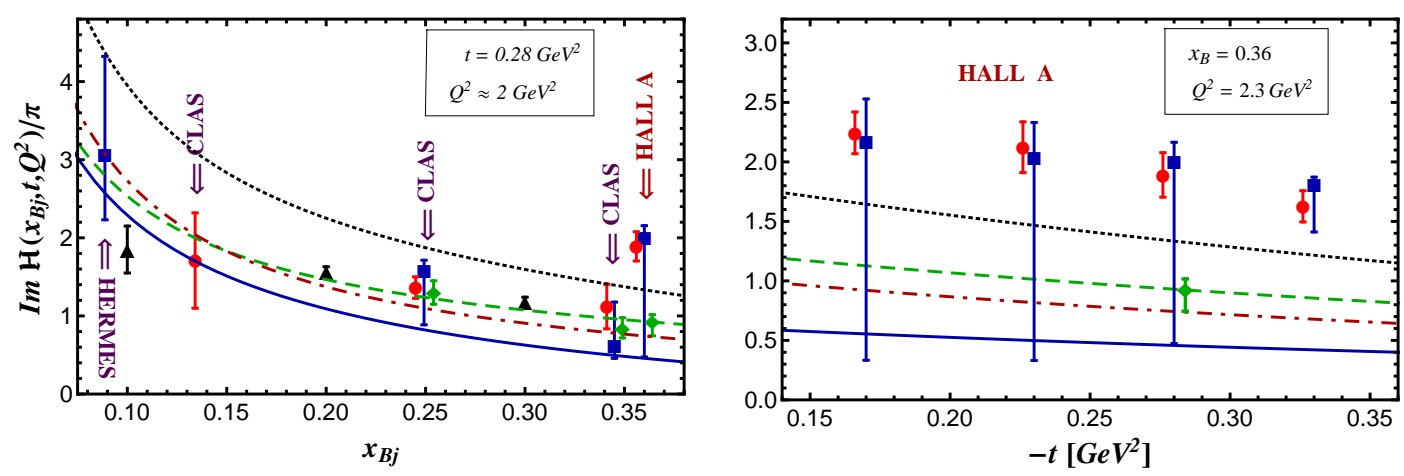

Figure 4: $\mathfrak{I m} \mathscr{H} / \pi$ obtained from different strategies: our DVCS fits [dashed (solid) curve excludes (includes) Hall A data from "dispersion relation" KM09a (KM09b) [ए5] and hybrid KM10b (dash-dotted) mod-

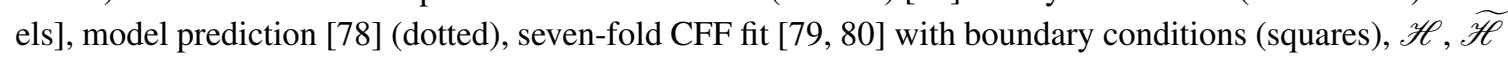
CFF fit [ [8]] (diamonds), smeared conformal partial wave model fit [82] within $H$ GPD (circles).

requires also the development of software tools. On the experimental side, new high-luminosity experiments with dedicated detectors are needed to get a deeper insight into both the underlying QCD mechanism and the exploration of the nucleon. Finally, we like to apologize that not all theoretical developments were even mentioned here, e.g., new non-perturbative quantities which are generalized distribution amplitudes (the crossed analog of GPDs) [55, [3] ] and transition distribution amplitudes [84] have been proposed for the description of two meson production via hard photon fusion and exclusive hard processes in hadron-hadron scattering experiments, respectively.

\section{References}

[1] M. Gell-Mann, Phys. Lett. 8 (1964) 214; G. Zweig, CERN-preprints Th. 401 and 412, 1964.

[2] E.D. Bloom et al., Phys. Rev. Lett. 23 (1969) 930.

[3] M. Breidenbach et al., Phys. Rev. Lett. 23 (1969) 935.

[4] R.P. Feynman, Phys. Rev. Lett. 23 (1969) 1415.

[5] J. Bjorken and E.A. Paschos, Phys. Rev. 185 (1969) 1975.

[6] H. Fritzsch, M. Gell-Mann and H. Leutwyler, Phys. Lett. B 47 (1973) 365.

[7] H. Politzer, Phys. Rev. Lett. 30 (1973) 1346.

[8] D. Gross and F. Wilczek, Phys. Rev. Lett. 30 (1973) 1343.

[9] K.G. Wilson, Phys. Rev. D 10 (1974) 2445.

[10] J.B. Kogut and L. Susskind, Phys. Rev. D 11 (1975) 395.

[11] M. Shifman, A. Vainshtein and V. Zakharov, Nucl. Phys. B 147 (1979) 385,448.

[12] S.D. Drell, D.J. Levy and T.M. Yan, Phys. Rev. Lett. 22 (1969) 744.

[13] S.D. Drell and T.M. Yan, Phys. Rev. Lett. 24 (1970) 181.

[14] D.S. Hwang and D. Müller, Phys. Lett. B660 (2008) 350 [arXiv:0710.1567].

[15] G.B. West, Phys. Rev. Lett. 24 (1970) 1206. 
[16] S.J. Brodsky and S.D. Drell, Phys. Rev. D 22 (1980) 2236.

[17] S.J. Brodsky, H.C. Pauli and S.S. Pinsky, Phys. Rept. 301 (1998) 299, hep-ph/9705477.

[18] A.H. Mueller (Ed.), Perturbative Quantum Chromodynamics, World Scinetific, Singapore, 1989.

[19] Y. Dokshitzer, Sov. Phys. JETP 46 (1977) 641.

[20] V. Gribov and L. Lipatow, Sov. J. Nucl. Phys. 15 (1972) 438.

[21] G. Altarelli and G. Parisi, Nucl. Phys. B 126 (1977) 298.

[22] S. Moch, J.A.M. Vermaseren and A. Vogt, Nucl. Phys. B 688 (2004) 101 [hep-ph/0403192].

[23] A. Vogt, S. Moch and J.A.M. Vermaseren, Nucl. Phys. B 691 (2004) 129 [hep-ph/0404111].

[24] S. Brodsky and G. Farrar, Phys. Rev. Lett. 31 (1973) 1153.

[25] V. Matveev, R. Muradyan and V. Tavkhelidze, Lett. Nuovo Cimento 7 (1973) 719.

[26] P. Landshoff, Phys. Rev. D 10 (1974) 1024.

[27] A. Efremov and A. Radyushkin, Phys. Lett. B 94 (1980) 245.

[28] G.P. Lepage and S.J. Brodsky, Phys. Lett. B 87 (1979) 359.

[29] G. Lepage and S. Brodsky, Phys. Rev. D 22 (1980) 2157.

[30] V. Chernyak and A. Zhitnitsky, Nucl. Phys. B 201 (1982) 492, (E) B 214 (1983) 547.

[31] N. Isgur and C.H. Llewellyn Smith, Phys. Rev. Lett. 52 (1984) 1080.

[32] A.V. Radyushkin, Acta Physica Polonica B15 (1984) 403.

[33] J. Botts and G. Sterman, Nucl. Phys. B 325 (1989) 62.

[34] H.N. Li and G. Sterman, Nucl. Phys. B 381 (1992) 129.

[35] I.I. Balitsky, V.M. Braun and A.V. Kolesnichenko, Nucl. Phys. B 312 (1989) 509.

[36] C.W. Bauer et al., Phys. Rev. D 63 (2001) 114020 [hep-ph/0011336].

[37] Jefferson Lab Hall A, M.K. Jones et al., Phys. Rev. Lett. 84 (2000) 1398 [nucl-ex/9910005].

[38] Jefferson Lab Hall A, O. Gayou et al., Phys. Rev. Lett. 88 (2002) 092301 [nucl-ex/0111010].

[39] V. Punjabi et al., Phys. Rev. C 71 (2005) 055202 [nucl-ex/0501018].

[40] A. Puckett et al., Phys.Rev.Lett. 104 (2010) 242301 [arXiv:1005.3419].

[41] A. Duncan and A. Mueller, Phys. Rev. D 21 (1980) 1636.

[42] A.V. Belitsky, X. Ji and F. Yuan, Phys. Rev. Lett. 91 (2003) 092003 [hep-ph/0212351].

[43] BaBaR coll., B. Aubert et al., Phys. Rev. D 80 (2009) 052002 [arXiv:0905.4778].

[44] BaBaR coll., P. del Amo Sanchez et al., 2011 [arXiv:1101.1142].

[45] A.P. Bakulev, S.V. Mikhailov and N.G. Stefanis, Phys. Rev. D 73 (2006) 056002 [hep-ph/0512119].

[46] S.V. Mikhailov and N.G. Stefanis, Nucl. Phys. B 821 (2009) 291 [arXiv:0905.4004].

[47] B. Melić, D. Müller and K. Passek-Kumerički, Phys. Rev. D 68 (2003) 014013 [hep-ph/0212346].

[48] A.V. Radyushkin, Phys. Rev. D 80 (2009) 094009 [arXiv:0906.0323].

[49] M.V. Polyakov, JETP Lett. 90 (2009) 228 [arXiv:0906.0538]. 
[50] A. Dorokhov, 2010 [arXiv:1003.4693].

[51] V. Chernyak, 2009 [arXiv:0912.0623].

[52] H.n. Li and S. Mishima, Phys. Rev. D 80 (2009) 074024, [arXiv:0907.0166].

[53] P. Kroll, 2010 [arXiv:1012.3542].

[54] S. Agaev et al., 2010 [arXiv:1012.4671].

[55] D. Müller et al., Fortschr. Phys. 42 (1994) 101 [hep-ph/9812448].

[56] A.V. Radyushkin, Phys. Lett. B 380 (1996) 417 [hep-ph/9604317].

[57] X. Ji, Phys. Rev. Lett. 78 (1997) 610 [hep-ph/9603249].

[58] J. Collins, L. Frankfurt and M. Strikman, Phys. Rev. D 56 (1997) 2982 [hep-ph/9611433].

[59] J. Collins and A. Freund, Phys. Rev. D 59 (1999) 074009 [hep-ph/9801262].

[60] J.P. Ralston and B. Pire, Phys. Rev. D 66 (2002) 111501 [hep-ph/0110075].

[61] M. Burkardt, Phys. Rev. D 62 (2000) 071503 [hep-ph/0005108]; (E) D 66 (2002) 119903.

[62] M.V. Polyakov and C. Weiss, Phys. Rev. D 60 (1999) 114017 [hep-ph/9902451].

[63] M. Diehl, Phys. Rept. 388 (2003) 41 [hep-ph/0307382].

[64] A.V. Belitsky and A.V. Radyushkin, Phys. Rept. 418 (2005) 1 [hep-ph/0504030].

[65] A.V. Radyushkin Phys. Rev. D56 (1997) 5524 [hep-ph/9704207].

[66] A.V. Belitsky, D. Müller and A. Kirchner, Nucl. Phys. B 629 (2002) 323 [hep-ph/0112108].

[67] O.V. Teryaev, 2005 [hep-ph/0510031].

[68] K. Kumerički, D. Müller and K. Passek-Kumerički, Nucl. Phys. B 794 (2008) 244 [hep-ph/0703179].

[69] M. Diehl and D.Y. Ivanov, Eur. Phys. J. C 52 (2007) 919 [arXiv:0707.0351].

[70] K. Kumerički, D. Müller and K. Passek-Kumerički, Eur. Phys. J. C 58 (2008) 193 [arXiv:0805.0152].

[71] A.V. Belitsky, A. Freund and D. Müller, Nucl. Phys. B 574 (2000) 347 [hep-ph/9912379].

[72] A.V. Belitsky and D. Müller, Phys. Lett. B 417 (1998) 129 [hep-ph/9709379].

[73] A.V. Belitsky and D. Müller, Phys. Lett. B 513 (2001) 349 [hep-ph/0105046].

[74] D.Yu. Ivanov, L. Szymanowski, and G. Krasnikov, JETP Lett. 80 (2004) 226 [hep-ph/0407207].

[75] K. Kumeriĉki and D. Müller, Nucl. Phys. B 841 (2010) 1 [arXiv:0904.0458].

[76] A. Donnachie, Phys. Lett. B 611 (2005) 255 [hep-ph/0412085].

[77] H1 coll., F.D. Aaron et al., Phys. Lett. B 681 (2009) 391 [arXiv:0907.5289].

[78] S.V. Goloskokov and P. Kroll, Eur. Phys. J. B C 53 (2008) 367 [arXiv:0708.3569].

[79] M. Guidal, Nucl. Phys. Proc. Suppl. B 184 (2008) 234 [arXiv:0803.1592].

[80] M. Guidal and H. Moutarde, Eur. Phys. J. B A 42 (2009) 71 [arXiv:0905.1220].

[81] M. Guidal, Phys. Lett. B 689 (2010) 156 [arXiv:1003.0307].

[82] H. Moutarde, Phys. Rev. D 79 (2009) 094021 [arXiv:0904.1648].

[83] M. Diehl, T. Gousse, B. Pire, and O.V. Teryaev, Phys. Rev. Lett. 81 (1998) 1782 [hep-ph/9805380].

[84] B. Pire and L. Szymanowski, Phys. Lett. B 622 (2005) 83 [hep-ph/0504255]. 